\title{
MEASURABILITY AND MODULARITY IN THE THEORY OF LATTICES ${ }^{1}$
}

\author{
M. F. SMILEY
}

In a previous note ${ }^{2}$ a notion of measurability (with respect to a function $\mu$ ) of elements of an arbitrary lattice was introduced. Our purpose there was to study closure properties of the subset of measurable elements. To do this it was convenient to assume that the lattice was modular. Results of V. Glivenko, ${ }^{3}$ and of L. R. Wilcox and the author ${ }^{4}$ indicate that the idea of measurability and that of modularity are intimately related. The purpose of this note is to exhibit a further relationship which does not depend on metric properties of the function $\mu$.

In a lattice $L$ we call the ordered pair $(a, b)$ of elements of $L$ modular and write $(a, b) M$ in case $\left(a_{1}+a\right) b=a_{1}+a b$ for every $a_{1} \leqq b$. This relation has been studied by $\mathrm{L}$. R. Wilcox. ${ }^{5}$ If $\mu(a)$ is a real valued function defined on $L$ we say that an element $c \in L$ is $\mu$-measurable in case

$$
\mu(c)+\mu(b)=\mu(c+b)+\mu(c b)
$$

for every $b \in L$. The symbol $L(\mu)$ will denote the totality of elements of $L$ which are $\mu$-measurable. We call $\mu$ proper in case $a \leqq b$ with $\mu(a)=\mu(b)$ implies $a=b$.

Before we discuss the general case, let us consider a lattice $L_{0}$ of finite dimension (that is, satisfying both chain conditions) in which every principal chain joining two elements $a, b \in L_{0}$ has the same length $n(a, b)$. Examples of such lattices are well known. ${ }^{6}$ Let 0 de-

1 Presented to the Society, February 24, 1940.

2 A note on measure functions in a lattice, this Bulletin, vol. 46 (1940), pp. 239-241. This concept specializes to that of permutability with all subgroups (that is, the quasi-normality of O. Ore, Structures and group theory I, Duke Mathematical Journal, vol. $3(1937)$, p. 162) if we set $\mu(s)=\log o(s)$ in the lattice of subgroups of a finite group, and to that of measurability in the sense of Carathéodory with respect to an outer measure function.

${ }^{3}$ Contributions à l'étude des systèmes de choses normées, American Journal of Mathematics, vol. 59 (1937), pp. 933-934.

4 Metric lattices, Annals of Mathematics, (2), vol. 40 (1939), p. 313.

5 Modularity in the theory of lattices, Annals of Mathematics, (2), vol. 40 (1939), p. $491 \mathrm{ff}$.

"The "exchange" lattices discussed by Saunders Mac Lane ( $A$ lattice formulation for transcendence degrees and p-bases, Duke Mathematical Journl, vol. 4 (1938), pp. 455-468) as well as the "semi-modular" lattices of finite dimension of Wilcox (op. cit., pp. 502-505) have these properties. We make no use, however, of the metric properties of these systems. 
note the least element of $L_{0}$ and define $\mu_{0}(a) \equiv n(0, a)$. We note that $\mu_{0}$ is proper. These considerations lead to the following theorem.

TheOREm 1. If $c \in L_{0}$ then $c_{1} \in L_{0}\left(\mu_{0}\right)$ for every $c_{1} \leqq c$ if and only if $a \leqq c$ implies that $(b, a) M$ for every $b \in L_{0}$ and furthermore that $x=a x+b$ for every $x \in L_{0}$ for which $b \leqq x \leqq a+b$.

Proof. To show the sufficiency of the condition consider elements $b, c_{1} \in L_{0}$ for which $c_{1} \leqq c$. It is easily seen that $c_{1} \in L_{0}\left(\mu_{0}\right)$ provided that $n\left(c_{1} b, c_{1}\right)=n\left(b, c_{1}+b\right)$. We shall prove in fact that $c_{1} / c_{1} b \simeq_{c_{1}}+b / b$. As usual, for $x_{1} \in c_{1} / c_{1} b$, that is, $c_{1} b \leqq x_{1} \leqq c_{1}$, define $f\left(x_{1}\right) \equiv x_{1}+b$. Clearly $f\left(x_{1}\right) \in c_{1}+b / b$. Also $f\left(c_{1} / c_{1} b\right)=c_{1}+b / b$; for if $b \leqq x \leqq c_{1}+b$, by hypothesis $f\left(c_{1} x\right)=x$. Suppose $f\left(x_{1}\right)=f\left(x_{2}\right)$, that is, $x_{1}+b=x_{2}+b$. Clearly $\left(x_{1}+b\right) c_{1}=\left(x_{2}+b\right) c_{1}$. By hypothesis $\left(b, c_{1}\right) M$, and it follows that $x_{1}+b c_{1}=x_{2}+b c_{1}, x_{1}=x_{2}$. Thus $f\left(x_{1}\right)$ establishes a one-to-one correspondence between $c_{1} / c_{1} b$ and $c_{1}+b / b$. This correspondence preserves the ordering relation and hence $c_{1} / c_{1} b \simeq c_{1}+b / b$. The proof of the sufficiency of the condition is complete. The proof of the necessity is included in the proof of the following theorem.

Theorem 2. Suppose that $\mu(a)$ is a proper real valued function defined on a lattice $L$. Consider an element $c \in L$. If $c_{1} \in L(\mu)$ for every $c_{1} \leqq c$, then $(b, a) M$ for every $a \leqq c$ and every $b \in L$, and furthermore $x=a x+b$ for every $x \in L$ such that $b \leqq x \leqq a+b$.

Proof. Consider elements $a, b \in L$ with $a \leqq c$. To show that $(b, a) M$, it suffices, since $\mu$ is proper, to prove that

$$
\mu\left(\left(a_{1}+b\right) a\right)=\mu\left(a_{1}+a b\right)
$$

for every $a_{1} \leqq a$. By hypothesis $a_{1}, a \in L(\mu)$ and consequently

$$
\begin{aligned}
\mu\left(\left(a_{1}+b\right) a\right) & =\mu\left(a_{1}+b\right)+\mu(a)-\mu(a+b) \\
& =\mu\left(a_{1}+b\right)+\mu(a b)-\mu(b) \\
& =\mu\left(a_{1}\right)+\mu(b)-\mu\left(a_{1} b\right)+\mu(a b)-\mu(b) \\
& =\mu\left(a_{1}\right)+\mu(a b)-\mu\left(a_{1} b\right)=\mu\left(a_{1}+a b\right) .
\end{aligned}
$$

Thus we have $(b, a) M$. Now consider an element $x \in L$ for which $b \leqq x \leqq a+b$. Clearly $a x \leqq a \leqq c$ and hence $a, a x \in L(\mu)$. It follows that

$$
\begin{aligned}
\mu(a x+b) & =\mu(a x)+\mu(b)-\mu(a b) \\
& =\mu(x)+\mu(a)-\mu(a+x)+\mu(b)-\mu(a b) \\
& =\mu(x)+\mu(a)+\mu(b)-\mu(a+b)-\mu(a b) \\
& =\mu(x) .
\end{aligned}
$$


Since $\mu$ is proper we have $x=a x+b$ and this completes the proof.

Corollary. If $p \in L$ is a point, then $p \in L(\mu)$ implies that $b+p$ is prime over $b$ for every $b \in L$ such that $b p=0$.

Proof. Consider a point $p \in L(\mu)$ and an element $b \in L$ for which $b p=0$. We always have $(b, p) M$ and thus the first part of our conclusion is vacuous. ${ }^{7}$ The second part yields $x=b+p x$ for every $x \in L$ for which $b \leqq x \leqq b+p$. Since $p x=0$ or $p x=p$, it follows immediately that $b+p$ is prime over $b$.

Remark. Consider the example of a lattice of six elements $0, p, q, b$, $c, 1$, with $p<c, q<b, 0$ the least element, and 1 the greatest element. Our corollary yields the fact that no proper real valued function $\mu$ can be defined over this lattice for which $p$ or $q$ is $\mu$-measurable.

LEHIGH UNIVERSITY

${ }^{7}$ L. R. Wilcox, op. cit., p. 491. 